
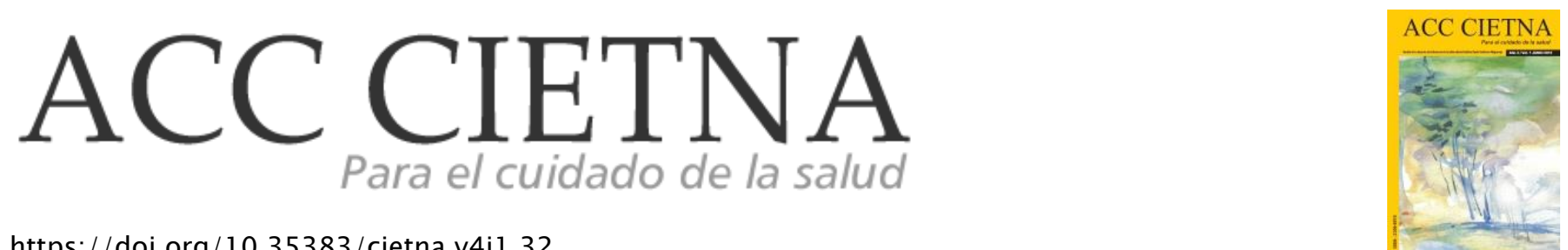

https://doi.org/10.35383/cietna.v4i1.32

\title{
Significados y atributos de la práctica de enfermería: una contribución para la enfermería geriátrica.
}

\author{
Castro Bellodas Mayra Alejandra ${ }^{1}$, Julcahuanca Balcázar Mariela Lisset ${ }^{2}$, Lázaro Alcántara \\ Elaine $^{3}$
}

\section{INFORMACIÓN DEL ARTÍCULO \\ Historia del artículo: \\ Recibido el 23 de septiembre de 2016 \\ Aceptado el 15 de diciembre de 2016}

\section{Palabras clave:}

Práctica

Enfermería

Sistematización

Geriátrica

\section{RESUMEN}

La presente investigación cualitativa, tuvo como objetivos: describir y analizar los significados y atributos de la práctica de Enfermería en el área de Geriátrica de un hospital, tomando como referencia la Clasificación internacional para la práctica de enfermería CIPE (1997). Participaron siete enfermeros, quienes poseían no menos de 3 años de experiencia en el área. Para el recojo de información se aplicó una entrevista semi estructurada, un test tipo Likert para la observación y data del participante. Los datos obtenidos fueron analizados con el método de análisis de contenido (Bardin, 2006), identificándose dos grandes categorías: Significados de la práctica de enfermería frente a los fenómenos fisiológicos y psicológicos del adulto mayor. Atributos de la práctica de enfermería frente al entorno del adulto mayor del adulto mayor. Se puede afirmar que desde la perspectiva de enfermería la sistematización del cuidado al adulto mayor se centra en la satisfacción de necesidades que van de lo fisiológico a lo ambiental. La Clasificación CIPE aplica de forma eficiente en la sistematización de la práctica de enfermería en el cuidado al adulto mayor otorgándole un orden común y organizándola, sin perder de vista la individualidad de la persona, visibilizando el actuar propio de enfermería.

\footnotetext{
${ }^{1}$ Licenciada. Enfermera con trabajo independiente, Chiclayo, Perú. Email: alecb_@hotmail.com

2 Licenciada. Enfermera con trabajo independiente, Chiclayo, Perú.

${ }^{3}$ Doctora. en Enfermería. Docente de Postgrado de la Universidad Católica Santo Toribio de Mogrovejo. Enfermera asistencial del Hospital de la Sanidad de la Policia. Chiclayo, Perú. Email: elazaro@usat.edu.pe
} 

nursing.

\section{ABSTRACT}

Keywords:

Practice

Nursing

Systematization

Geriatrics.
The present qualitative investigation, it had as aims: to describe and to analyze the meanings and attributes of the practice of Infirmary in the area of Geriatric of a hospital, taking the international Classification as a reference to the practice of infirmary CIPE (1997). There took part seven nurses, who were possessing not less than 3 years of experience in the area. For I gather of information an interview was applied semi structured, a test type Likert for the observation and byline of the participant. The obtained information was analyzed by the method of analysis of content (Bardin, 2006), Two big categories being identified: Meanings of the practice of infirmary opposite to the physiological and psychological phenomena of the major adult. Attributes of the practice of infirmary opposite to the environment of the major adult of the major adult. It is possible to affirm that from the perspective of infirmary the systematizing of the care centres the major adult on the satisfaction of needs that go from the physiological thing to the environmental thing. The Classification CIPE applies of efficient form in the systematizing of the practice of infirmary in the care the major adult granting a common order to him and organizing it, without losing of sight the individuality of the person, visibilizando to act own of infirmary.

\section{Introducción}

La práctica de enfermería ha ido desarrollándose de forma progresiva, basando sus actividades en conocimientos y técnicas que han experimentado un progreso considerable, a lo largo del tiempo, generando a la vez cambios en la sistematización del cuidado'.

Estas actividades tienden a transformarse bajo el efecto del desarrollo tecnológico, sin olvidar el impacto de fenómenos como: el proceso de envejecimiento de la población, la diversidad cultural y los cambios en el cuidado, lo que genera nuevas demandas que reconfiguran la razón de ser de su profesión. Este descentramiento en la razón de ser de la profesión tiene su origen desde mucho tiempo atrás, ya que la enfermería se ha preocupado siempre por evidenciar las prácticas de cuidado, a través del desarrollo de modelos y teorías cuya pionera fue Florence Nightingale, quien heredó a enfermería un constructo de conceptos relacionados con la salud, entorno, persona y el cuidado2, 3, 4.

Frente a esta realidad aparecen los primeros escritos de enfermería sobre el proceso, asumiendo que éste sería la piedra angular de la práctica de enfermería. A mediados de 1995, cuando Lidia Hall / describía la enfermería como un proceso, sin embargo, su descripción tardó varios años en tener aceptación. Posteriormente en 1967, Yura y Walsh crearon un primer texto que describía un proceso con cuatro fases: valoración, planeación, ejecución y evaluación. Blodi en 1974 y Roy en 1975 añadieron la etapa de diagnóstico dando lugar a un proceso de cinco fases ${ }^{3}$.

La lucha de las enfermeras por definir el campo de la profesión y con el objetivo de obtener un status profesional enfocó sus investigaciones hacia el desarrollo del diagnóstico de enfermería. Se creó entonces la taxonomía NANDA en 1973, que en su trayecto se ha detectado que carece de un enfoque filosófico lógico y de unas competencias 
claramente definidas que delimiten claramente qué es y qué no es un juicio diagnóstico enfermero; incluso en la taxonomía de situaciones que difícilmente pueden ser identificadas $y$ tratadas independientemente por las enfermeras, al haber eliminado la diferencia entre las características definitorias en críticas mayores y menores todas tienen el mismo peso específico a la hora de identificar un diagnóstico.

Siguiendo esta línea, surge la Clasificación internacional para la Práctica de la Enfermería (CIPE) en 1991, como respuesta a la necesidad de sistematizar las actividades de enfermería, la cual fue reconocida, hace más de una década, por el Consejo Internacional de Enfermeras (CIE), con la finalidad de crear un vocabulario de términos estructurado para describir y organizar la práctica de enfermería ${ }^{5,6}$.

Frente a la realidad descrita se formula la siguiente interrogante de investigación: ¿Cómo contribuyen los significados y atributos de la práctica de enfermería Geriátrica desde la perspectiva de la enfermera? El objeto de estudio se definió como: contribución de los significados y atributos de la práctica de Enfermería. Los objetivos que se plantearon en esta investigación fueron: Describir los significados y atributos de la práctica de enfermería como contribución para la Geriátrica desde la perspectiva de la enfermera. Analizar estos significados y atributos de la práctica de enfermería Geriátrica.

En la actualidad la práctica de enfermería en los servicios de geriatría se basan en actividades rutinarias como administración de medicamentos, curación de heridas entre otros, haciendo que el cuidado no tenga una secuencia sobre el servicio que se brinda. . Es por eso que en esta investigación se pretendió mediante el CIPE que las enfermeras de este servicio tengan un lenguaje común, donde la evidencia del cuidado sea más dinámica, partiendo de la sistematización de su práctica cotidiana de cuidado al adulto mayor. La contribución del presente trabajo se configura en los resultados obtenidos que han permitido sistematizar en forma ordenada las actividades desarrolladas por enfermería, dando a su práctica orden y un lenguaje común, para la aplicación del cuidado al adulto mayor, de los elementos inmersos en la clasificación CIPE aplicados al cuidado del adulto mayor; contribuyeron con la visibilización del trabajo del profesional de enfermería en geriatría, el cual carecía de estándares de medición, siendo difícil mostrarlo, y a partir de los resultados se puede afirmar cuales son los fenómenos de cuidado que ocupan el trabajo del profesional de enfermería. Los resultados se convierten en una posibilidad de sentar una línea de investigación dentro del cuidado, que permita seguir sistematizando la práctica de cuidado desarrolladas por las enfermeras, fortaleciéndola como una práctica basada en la evidencia, tan escasa en nuestra realidad.

\section{Metodología}

La investigación es de tipo cualitativo, siendo abordada metodológicamente por el estudio de caso, que permitió conocer a profundidad a los sujetos. Este abordaje metodológico permitió conocer aquellos factores que influyen sobre la práctica de enfermería, dándole una visión holística al problema estudiado, lo cual es muy característico del estudio de caso ${ }^{7,8,9}$.

El caso fue abordado en dos grandes momentos, el primer momento donde se hizo una revisión exhaustiva de la literatura de tal manera que se comprenda el objeto de estudio y ver de qué manera han sido enfocado a las diferentes investigaciones, ya en un segundo momento se pasa a la discusión, donde se evaluó que caminos debemos seguir, desde el momento de establecer los objetivos hasta llegar a las conclusiones.

Para el desarrollo del segundo momento se tienen en cuenta las siguientes fases: Definir la unidad de caso; aquí las investigadoras requirieron de ciertas habilidades para recopilar y entender los datos suficientes, llegando a una comprensión más detallada del objeto como un todo. La recopilación de datos; en este caso se trabajó con instrumentos cualitativos y cuantitativos, entre los cuales se describen los siguientes: la entrevista 
con preguntas abiertas a profundidad y una lista de cotejo, permitiendo obtener información necesaria. La selección análisis e interpretación de datos; para la selección de datos se consideraron los objetivos de la investigación, además de un sistema de referencia para evaluar si los datos son útiles o no, en este caso los analizamos con los datos obtenidos, para verificar que estos respondan a los objetivos de la investigación. En la última fase se prepararon los informes parciales y finales, recordando que los datos Obtenidos fueron específicos, que las teorías.

Utilizadas y las categorizaciones que se obtuvieron finalmente demuestren la validez y fiabilidad de la investigación. Los participantes en la investigación fueron ocho Enfermeros, las cuales fueron seleccionadas por conveniencia.

El escenario fue, el servicio de Geriatría del Hospital Nacional Almanzor Aguinaga Asenjo, un hospital de tercer nivel de atención, ubicado en el departamento de Lambayeque, provincia de Chiclayo, fue en este hospital donde se inició por primera vez el servicio de Geriatría. Se escogió este servicio por la gran demanda de atención que se presenta con los adultos mayores, en donde en su mayoría se vuelven parcialmente o totalmente dependiente del cuidado enfermero.

El estudio requirió de diferentes instrumentos de recolección de datos como: una lista de cotejo elaborada de acuerdo al CIPE y la entrevista semiestructurada. Se hicieron dos listas de cotejos, la primera para las enfermeras, quienes registraron a su criterio las actividades que cumplían según el CIPE entorno a las prácticas del adulto mayor, La otra lista de cotejo fue llenada por las investigadoras, que observaron el actuar de las enfermeras. Ambos instrumentos siguieron la misma estructura pero lo que direcciono fue los puntos de observación, se contó con escalas de medición fueron del $0-3$ donde cero significa "nunca ( 0 veces en el turno)", uno "casi nunca ( 1 vez en el turno)", dos "algunas veces (2 veces en el turno)" y tres "siempre (3 o más veces en el turno)". La entrevista se estructuró con preguntas abiertas a profundidad que exploraron los fenómenos considerados en el CIPE. La entrevista fue aplicada en su mismo escenario laboral con el consentimiento informado del participante, permitiendo abordar de manera profunda cada una de las unidades de análisis, así mismo, se aplicó observación a las enfermeras corroborando sus actividades cotidianas, utilizando una lista de cotejo siguiendo la lógica CIPE.

La investigación se rige por los principios de bioética personalista, que se fundamenta en el respeto al valor de la vida y a la dignidad de la persona; para ello se inició el contacto con los participantes informándoles sobre los objetivos, el objeto de la investigación, se le proporcionó para su lectura el formato del consentimiento informado, donde se configura a que tiene derecho, se les informo que podrían retirarse en cualquier momento del estudio, si consideraban que no deberían contestar una pregunta no la contestaban.

La recopilación de datos se realizó de una manera libre, sin presión alguna, y con previa información del procedimiento que se iba a realizar. Entre los criterios aplicados en esta investigación se encuentran el principio de libertad $y$ responsabilidad, el principio de sociabilidad ${ }^{10,11}$. Los criterios de rigor científico utilizados en la investigación fueron el de Credibilidad, confirmabilidad o auditabilidad, transferibilidad o aplicabilidad, dependencia o consistencia. En el último criterio que es la triangulación, se pretendió realizar una posición específica respecto a los resultados obtenidos mediante la utilización de los instrumentos cualitativos y cuantitativos para la recolección de datos, los cuales posteriormente permitieron realizar un análisis más detallado, haciendo comparaciones para conocer de mejor manera la realidad de la práctica de enfermería en el adulto mayor, tomando como puntos finales las conclusiones y consideraciones de esta investigación ${ }^{12}$.

Según estos criterios se trataron los resultados de la investigación, manejando dos tipos de resultados cuantitativos y cualitativos, los que han sido analizados con la triangulación de datos 
obteniendo los siguientes Los resultados cuantitativos fueron analizados en conjunto con los resultados cualitativos obtenidos de la entrevista semi-estructurada, con la finalidad de responder al criterio analítico del estudio de caso.

\section{Resultados, análisis y discusión}

Las características de la respiración y permeabilidad de las vías aéreas son valoradas en un $71 \%$ algunas veces, la actividad en movimiento $y$ en reposo un $57 \%$ algunas veces, en el grafico paralelo se observa diferencia entre los 2 primeros ítems características de la respiración y permeabilidad ya que registran un $71 \%$ siempre, indicando que las enfermeras consideran los 4 parámetros que propone el CIPE para valorar este fenómeno, reflejándose la importancia que otorgan a esta necesidad, por ser considerada como fundamental para la supervivencia del ser humano. Analizando la información con el discurso correspondiente de la entrevista, se identifica que las enfermeras consideran esta necesidad como unas de las prioritarias para el cuidado del adulto mayor al igual que el CIPE, sin embargo ellas controlan la saturación de oxigeno dentro de esta necesidad, siendo un aporte para el CIPE para que esta necesidad sea valorada por completo, en beneficio de la salud del adulto mayor. Se identifica el control de la frecuencia cardiaca un $57 \%$ siempre y el control de la presión arterial un $43 \%$ siempre, sin embargo el grafico paralelo nos muestra un control en un $71 \%$ en ambos ítems, siendo los porcentajes muy distantes de lo referido por las enfermeras a la realidad observada por las investigadoras, definiéndose de esta manera que cuando la necesidad esta alterada incrementa el número de frecuencia en que es valorada, triangulando esta información con el discurso obtenido de la entrevista, se identifica que las enfermeras ponen más énfasis en valorar esta necesidad cuando se presenta una alteración, aumentando su frecuencia y presión arterial y por ende su control es indispensable. En las características de la frecuencia cardiaca las enfermeras refieren que solo consideran algunas, siendo este el aporte del CIPE para que esta necesidad sea valorada por completo en beneficio del adulto mayor.

Las enfermeras controlan la termorregulación en un $43 \%$ siempre y un $43 \%$ algunas veces, en el grafico paralelo se muestra un control de la termorregulación en un $57 \%$ algunas veces y un $43 \%$ casi nunca, identificando la gran diferencia entre los porcentajes obtenidos entre la lista llenada las enfermeras y la lista de las investigadoras, analizando estos resultados con lo obtenido en la entrevista se evidencia que la práctica está orientada a la resolución de cada una de estas cuando se produzca una alteración, actividades que no considera el CIPE, pero que se pueden realizar para cumplir con las demandas de los adultos mayores.

Las enfermeras controlan el peso en un $14 \%$ siempre, la talla y el IMC en un $29 \%$ casi nunca, la succión, masticación y deglución $43 \%$ siempre, en el grafico paralelo se observa que el control del peso, talla e IMC es de $0 \%$ nunca, succión, masticación y deglución un $57 \%$ casi nunca, identificando diferencia entre los porcentajes reflejando que las enfermeras muy poco priorizan la valoración de esta necesidad enfocándose más a la resolución cuando esta alterada, estos resultados más el discurso correspondiente obtenido en la entrevista, se identifica un gran porcentaje de diferencia en la valoración de esta necesidad, sin embargo la enfermera es quien identifica la alteración y trabaja en conjunto con diferentes interdisciplinas como la nutricionista quien evalúa estos parámetros que propone el CIPE para esta necesidad y es la enfermera quien evalúa cómo va la recuperación y que cuidados debe tener durante su jornada de trabajo.

Se observa que las enfermeras valoran la frecuencia de la eliminación urinaria en un $43 \%$, cantidad, color y olor en un $57 \%$ a diferencia de lo obtenido por las investigadoras donde se observa que la frecuencia y la cantidad en un $29 \%$ siempre, el color en un $43 \%$ y el olor un $29 \%$, evidenciándose diferencia entre los porcentajes que indican que las enfermeras si consideran los ítems que 
propone el CIPE sin embargo no todos con el mismo nivel importancia según lo reflejado en los gráficos, al triangular esta información con el discurso correspondiente obtenido de la entrevista, se identifica un estrecho margen de diferencia, lo que refleja que la enfermera evalúa esta necesidad, sin embargo la frecuencia es determinada si la persona presenta una alteración, afirmando lo mencionado en el párrafo anterior, dando el aporte del cipe en la valoración de las características de esta necesidad.

Se observa que la enfermeras evalúan los cuatro parámetros que propone el CIPE en un 57\% siempre, seguida de olor en un $14 \%$ algunas veces, en el grafico paralelo las investigadoras observaron que las enfermeras evalúan con mayor frecuencia, cantidad y color de las heces en un $57 \%$, para luego continuar con la frecuencia, cantidad y color de estas representado en $14 \%$ que siempre, triangulando esta información se reconoce un estrecho margen de diferencia entre ambos gráficos, lo que refleja que la enfermera evalúa esta necesidad, sin embargo la frecuencia es determinada si la persona presenta una alteración o no de esta necesidad, para realizar sus cuidados.

Se identifica que las enfermeras valorar la hidratación en un $57 \%$, la integridad en un $43 \%$, la turgencia en un $57 \%$ y la presencia de edema en un $71 \%$ siempre, la hidratación, integridad, turgencia y edema en un $29 \%$, observando un estrecho margan de deferencia, indicando que para las enfermeras es prioritaria esta necesidad con todo los parámetros que propone el CIPE, cruzando esta información con el discurso de la entrevista se identifica que las enfermeras consideran esta necesidad como prioritaria de valorar en los adultos mayores, debido a los cambios fisiológicos que se generan en la piel producto del proceso de envejecimiento, considerando los mismos aspectos que propone el CIPE para valorar esta necesidad, sin embargo ponen más énfasis en algunos, proponiendo el CIPE el mismo valor para todos los ítems que se valora en esta necesidad.
Además se observa que las enfermeras valoran todo ítems que propone el CIPE para este fenómeno, en el grafico paralelo se evidencia que las enfermeras valorar la necesidad descanso y sueño en un $57 \%$ casi nunca los factores que interrumpen el sueño en un $78 \%$ casi nunca, existiendo diferencia entre los porcentajes reflejándose que las enfermeras consideran 2 características que propone para valorar esta, al analizar estos resultados con el discurso de la

Entrevista identificamos diferencia en los porcentajes, reflejando que la enfermera evalúa esta necesidad, sin embargo la frecuencia es determinada si presenta una alteración o no, planificando sus cuidados en su jornada de trabajo, ya que en su rutina normal solo considera dos de los ítems que propone el CIPE.

Se identifica que las enfermeras valoran la fuerza y el tono y capacidad de movimiento en un $43 \%$ siempre, a diferencia de lo obtenido en el grafico siguiente donde se obtiene que la fuerza y la capacidad de movimiento son valorados en un $14 \%$ siempre, observando diferencia entre los porcentajes lo que refleja que la enfermera valora dos de los ítems que propone el CIPE para este fenómeno, analizando esta información con el discurso obtenido de la entrevista, identificamos un estrecho margen de diferencia, lo que refleja que la enfermera evalúa esta necesidad, sin embargo la frecuencia es determinada si la persona presenta una alteración para que esta sea evaluada en su jornada de trabajo, variando su frecuencia de valoración, siendo también un aporte para el CIPE, las actividades que si consideran las enfermeras importante en esta necesidad.

Comparando estos resultados obtenidos por la observación de las investigadoras, que en este caso el $29 \%$ valora la presencia del dolor, las características y la intensidad del dolor, seguido de un $14 \%$ que refiere valorar los medios para aliviar, las sensaciones y el estado de conciencia siempre, a diferencia de lo que refieren donde señalan que el $71 \%$ valora la presencia del dolor siempre seguida de un $57 \%$ que refiere valorar las 5 características restantes siempre, al confrontar 
estos resultados con los obtenidos en la entrevista se reconoce que las enfermeras orientan su práctica a la resolución de la alteración de esta necesidad, siendo una de las principales necesidad a resolver para el bienestar del adulto, dejado muchas veces de lado la valoración de algunos parámetros que el CIPE consideras importantes de valorar muy aparte de lo que la enfermera evalúa continuamente para dar resolución a esta necesidad, es por eso que el aporte del CIPE frente a este fenómeno en la evaluación de estos parámetros considerados en esta clasificación.

\begin{tabular}{|c|c|c|}
\hline \multicolumn{2}{|c|}{ FENOMENOS DEL CIPE } & ACTIVIDADES DE \\
\hline \multirow{9}{*}{$\begin{array}{l}\text { Funciones } \\
\text { Fisiológicas }\end{array}$} & $\begin{array}{l}\text { - Respiración. } \\
\text { - Permeabilidad de las } \\
\text { vías aéreas. }\end{array}$ & $\begin{array}{l}\text { Valoración de la } \\
\text { necesidad de respiración }\end{array}$ \\
\hline & $\begin{array}{l}\text { - Intolerancia a la } \\
\text { actividad. }\end{array}$ & $\begin{array}{l}\text { Valoración de la } \\
\text { necesidad de movimiento }\end{array}$ \\
\hline & $\begin{array}{l}\text { - Frecuencia cardiaca. } \\
\text { - Presión arterial. }\end{array}$ & $\begin{array}{l}\text { Valoración de la función } \\
\text { circulatoria. }\end{array}$ \\
\hline & - Termorregulación & $\begin{array}{l}\text { Valoración de la necesidad } \\
\text { de termorregulación }\end{array}$ \\
\hline & Nutrición & $\begin{array}{l}\text { Valoración de la necesidad } \\
\text { de alimentación. }\end{array}$ \\
\hline & $\begin{array}{l}\text { - Eliminación urinaria } \\
\text { - Eliminación intestinal. }\end{array}$ & $\begin{array}{l}\text { Valoración de la } \\
\text { necesidad de eliminación. }\end{array}$ \\
\hline & - Piel. & $\begin{array}{l}\text { Valoración de la necesidad } \\
\text { de integridad de la piel }\end{array}$ \\
\hline & - Descanso y sueño & $\begin{array}{l}\text { Valoración de la necesidad } \\
\text { de descanso y sueño. }\end{array}$ \\
\hline & Actividad física. & $\begin{array}{l}\text { Valoración de la } \\
\text { necesidad de movimiento. }\end{array}$ \\
\hline \multirow[t]{2}{*}{$\begin{array}{l}\text { Funciones } \\
\text { Psicológicas }\end{array}$} & Sensaciones. & $\begin{array}{l}\text { Valoración de la } \\
\text { necesidad de dolor }\end{array}$ \\
\hline & $\begin{array}{l}\text { - Estima y autoestima. } \\
\text { - Autocuidado. }\end{array}$ & $\begin{array}{l}\text { Valoración de la necesidad } \\
\text { estima y autoestima. }\end{array}$ \\
\hline
\end{tabular}

Comparando estos resultados obtenidos por la observación de las investigadoras, se obtiene que las únicas características que son valoradas con mayor frecuencia son la autoestima y el respeto a las creencias en un $14 \%$ siempre, lo cual contradice a lo referido por las enfermeras en donde nos indican que valoran con mayor frecuencia el temor del adulto en un 43\%, seguido de la valoración del autoestima, la ansiedad culpa y vergüenza, no reflejando en lo observado por las investigadoras, dando a entender la poca importancia que en algunos casos le otorgan para la valoración de este fenómeno, al confrontar estos resultados con lo obtenido en la entrevista se puede reconocer que las enfermeras orientan su práctica a la resolución de la alteración de esta necesidad, en conjunto con los familiares, siendo estos lo que continuaran con el cuidado en el hogar, pero además la enfermera es capaz de reconocer el estado de ánimo del adulto así como el respecto a sus creencias y costumbre, actividades que si son realizadas por las enfermeras y las cuales también son consideradas por el CIPE, para el bienestar del adulto, al triangular se identifica diferencia entre los porcentajes, sin embargo las enfermeras trabajan en conjunto con otras interdisciplinas, es este caso el profesional en psicología donde los casos son derivados y las enfermeras evalúan el progreso y ejecutan sus propias actividades en su jornada de trabajo.

Comparando estos resultados obtenidos por la observación de las investigadoras, que este caso es representado por el $14 \%$ quienes valoran algunas veces estos tres parámetros del autocuidado, no guardando similitud con lo referido por las enfermeras donde se obtiene que el $57 \%$ valoran siempre la higiene y la alimentación seguida de un $43 \%$ que valoran la vestimenta siempre, resultados que contradicen lo referido por ellas, en donde se evidencia que solo algunas veces toman en cuenta esta necesidad, reflejándose la poca importancia que en algunos casos se otorga para la valoración de este fenómeno psicológico, al triangular esta información, identificamos que las enfermeras consideran el Autocuidado como una de las necesidades prioritarias, donde es evaluada durante su jornada de trabajo y según su criterio establecen la frecuencia en que es reevaluada para identificar si la necesidad está siendo satisfecha. Aportando también al CIPE, el trabajo que ellas realizan al integrar al cuidador en el cuidado, para que este sea continúo.

Al final del análisis de todos los cuadros obtenidos se pudo realizar un cuadro donde se hace un paralelo de las actividades de Enfermería con los fenómenos que propone el CIPE, donde se observa 
que estas actividades están consideradas en esta clasificación, con diferentes denominaciones y actividades agregadas que complementen la valoración de estos fenómenos, siendo para las investigadoras importante organizar dicha información obtenida en la recogida de datos y establecer similitudes con la propuesta del CIPE, surgiendo así el paralelo presentado anteriormente donde refleja las actividades que realiza la enfermera con lo que propone el CIPE.

En tratamiento de datos cualitativos respalda los significados de la práctica de enfermería, teniendo en cuenta el tipo de tratamiento de datos (Método de asociación de contenidos), se sintetiza en el número de testimonio de las enfermeras que respaldaron cada categoría obtenida, presente a continuación.

Categoría I: Los significados de la práctica de enfermería frente a los fenómenos fisiológicos y psicológicos del adulto mayor.

1.1. El aspecto fisiológico centro de la práctica de enfermería

Kozzier, afirma que las necesidades fisiológicas son las que se relacionan con la supervivencia del individuo, es decir son las primordiales, básicas, esenciales y las más importantes de satisfacer, ya que el ser humano las necesita para sobrevivir, y se encuentran dentro de estas necesidades: respiración, frecuencia cardiaca, presión arterial, temperatura, alimentación, eliminación, movimiento, descanso y sueño, necesarias para la homeostasis, es decir para mantener un adecuado funcionamiento del organismos ${ }^{13}$.

Ante lo expuesto anteriormente y comparándolo con la información obtenida a través de las entrevistas, las enfermeras manifiestan.

“...priorizas tus actividades de acuerdo al grado de tus pacientes. En las necesidades fisiológicas las prioritarias son la respiración, la frecuencia cardiaca, valoramos muy puntualmente su temperatura y la presión arterial...". (Trabajadora de salud).

Este párrafo sustenta lo mencionado por el autor, en donde las enfermeras del servicio de geriatría, indican la importancia de priorizar las necesidades fisiológicas de acuerdo al grado de dependencia en que se encuentren los adultos mayores, señalando incluso algunas más importantes que otras, la cuales se deben tener en cuenta durante la valoración, de manera que respondan a la demanda de satisfacción de necesidades de esta población, siendo estas muy dependientes del cuidado enfermero.

La respiración es la primera necesidad fundamental de todo ser humano, definiéndose como el intercambio de oxígeno y $\mathrm{Co} 2$ que ocurre entre la atmósfera y las células del organismo, la ausencia de oxígeno conduce a la muerte, generalmente en este proceso participan dos sistemas el sistema pulmonar y el sistema cardiovascular, los cuales favorecen el intercambio gaseoso para el desarrollo de las necesidades metabólicas. Existen diversos factores que alteran esta función interrumpiendo la capacidad del organismo para satisfacer sus demandas de oxígeno, destacando principalmente la fase del desarrollo del ser humano específicamente del adulto mayor 13,14

Ante lo expuesto anteriormente y comparándolo con la información obtenida a través de las entrevistas, las enfermeras manifiestan.

“...en la respiración, medimos la saturación de oxígeno, observamos si esta con oxígeno, máscara de reservorio y si evoluciona correctamente cambiamos a Ventury, según su condición hasta cánula nasal, hasta que logre ventilar por sí solo, tenemos que actuar inmediatamente para evitar complicaciones y no esperar que el médico de la orden...". (Cuidadora del adulto).

La temperatura corporal es una necesidad fisiológica y prioritaria de resolver definiéndose como la diferencia entre la cantidad de calor producido por los procesos corporales y la cantidad de calor perdida al ambiente externo. Conocer la fisiología de la regulación de la temperatura corporal ayuda a la enfermera a valorar la respuesta del paciente a las alteraciones de la temperatura y a intervenciones de forma segura, se puede evidenciar en estos argumentos 
la capacidad de estas para solucionar las problemas que puedan surgir producto de este fenómeno 14.

"...en la temperatura acudimos primero por medios físicos, y si no se dan resultados positivos tenemos que ponerle su tratamiento antipirético, aligerarle cubiertas, educar al familiar...". (Trabajadora de salud). La nutrición es un elemento básico de todos los aspectos de la salud, esencial para las personas geriátricas, así como para la recuperación de la salud después de una enfermedad. El organismo necesita alimentos que le proporcione energía para el buen funcionamiento de los órganos, el movimiento corporal y las actividades de la vida diaria para mantener la temperatura corporal 13 .

Ante lo expuesto anteriormente y comparándolo con la información obtenida a través de las entrevistas, las enfermeras manifiestan.

“...en la necesidad de nutrición, la valoramos si el paciente esta desnutrido, anémico, mastica, deglute, evaluamos y reportamos al médico, para poder colocar una sonda nasogástrica, vemos que consuma toda su dieta, y se finaliza con la valoración de la sonda colocada, el tiempo de cambio, la alimentación, educación al familiar o cuidador, se coordina con nutrición, el peso del paciente, su contextura, supervisamos que consuma su dieta..." (Licenciada)

La eliminación urinaria es un proceso que la mayoría de personas dan por hecho cuando el aparato urinario funciona correctamente, ocasionando alteración cuando este deja de funcionar normalmente, pudiendo verse afectados todos los sistemas corporales, sobre todo en los adultos mayores cuando presentan alteración de esta necesidad tienden a experimentan problemas diversos relacionados con su imagen corporal(13,14). “...eliminación urinaria verificamos el tipo sonda para realizar el cambio respectivo, considerar a los pacientes que tiene retención urinaria, si usan sonda, presencia de pañal o colector, verificamos si existe alteración dermatológica como las escaldaduras, cambiando oportunamente el pañal...". (Cuidadora).

Otros de los principales aspectos del cuidado de enfermería radica en el mantenimiento de la integridad de la piel, la cual puede verse alterada o causada por una presión prolongada, irritación de la piel e inmovilidad, uno de los cuidados radica en permitir la detección precoz por parte de la enfermera en los paciente con mayor riesgo de desarrollar ulceras por decúbito, una vez identificado se tomaran las medidas necesarias para conservar la integridad de la piel 14 .

Ante lo expuesto anteriormente y comparándolo con la información obtenida a través de las entrevistas, las enfermeras manifiestan.

“...en las necesidades fisiológicas, valoramos la integridad de la piel, porque se pierde la hidratación, volviéndose más propensa a una lesión, eso es lo principal en nuestro adulto mayor...". (Enfermera).

El descanso y sueño son básicos para la calidad de vida de los adultos mayores, las distintas personas necesitan tipos y cantidades diferentes de sueño, sin sueño y descanso la capacidad de concentración y de participar en las actividades cotidianas disminuye, al tiempo que aumenta la irritabilidad, es por eso que el profesional de enfermería debe ocuparse de aquellas alteraciones preexistentes del sueño, como de aquellos que desarrollan problemas para dormir a consecuencias de otras alteraciones de la salud ${ }^{14}$.

Ante lo expuesto anteriormente y comparándolo con la información obtenida a través de las entrevistas, las enfermeras manifiestan.

“...también valoramos la necesidad de sueño, si un paciente no puede por la mala posición..." (Trabajadora de la salud)

La movilidad es la capacidad de una persona de moverse con libertad, se considera como el resultado de la interacción entre los aspectos 
físicos y psicosociales del paciente, aquellos pacientes con una movilidad completa pueden atender a sus necesidades por sí mismo o con la más mínima ayuda por parte de la enfermera, la falta de movilidad puede tener como consecuencia la inactividad física o la restricción física de los movimientos producto del reposo en cama, causando una reducción del movimiento corporal13,14.

Ante lo expuesto anteriormente y comparándolo con la información obtenida a través de las entrevistas, las enfermeras manifiestan.

“...Cuando esta alterado le Necesidad de movimiento lo movemos al borde la cama o lo bajamos a la silla de ruedas, lo que cuesta es subirlo a la cama, demanda de mayor esfuerzo físico, para nosotras muchas veces los dejamos sentados por qué no podemos subirlo..." (Cuidadora)

\subsection{Actividades propias de las enfermeras.}

El cuidado de las personas mayores exige más tiempo y dedicación ya que representan el importante núcleo de población con necesidades peculiares y específicas, en los cuales se enfocan las prácticas de enfermería. El cuidado de los ancianos es una actividad que está representando por los profesionales de enfermería, los cuales junto con el equipo multidisciplinario se encarga de la asistencia global e integral de los ancianos. Ante lo expuesto anteriormente y comparándolo con la información obtenida a través de las entrevistas, las enfermeras manifiestan.

“...en el turno diurno recibes el reporte, ordenas el material que se va necesitar para el tratamiento endovenoso $y$ tratamiento oral, administras medicamento de acuerdo al kardex, se van supervisando las vías, tratamientos, sondas nasogástricas, sonda Foley, preparación del material para curaciones de ulceras observamos si hay una buena higiene de genitales, se educa a la familia, tratamiento endovenoso, curación de ulceras por presión..." (Trabajadora de salud)

1.3. Práctica de enfermería enfocada en el aspecto psicológico.

Esta Sub categoría ha sido construida en base a testimonios de las enfermeras que laboran en un servicio de geriatría, reflejando la percepción que estas obtienen durante sus horas de trabajo. Entre las necesidades psicológicas mencionadas por las enfermeras tenemos: sensaciones, estima $y$ autoestima, Autocuidado. Las necesidades psicológicas es uno de los aspectos más importantes en el adulto mayor, ya que deben satisfacerse a fin de preservar la salud mental.

Ante lo expuesto anteriormente y comparándolo con la información obtenida a través de las entrevistas, las enfermeras manifiestan.

“...en las necesidades psicológicas, en el adulto mayor encontramos con frecuencia la depresión, soledad, los pacientes muchas veces están abandonados, hay maltrato verbal, conflictos familiares, entonces llamamos a la asistenta social, el apoyo espiritual que lo recibe del sacerdote que viene a visitarlos, el familiar también está ansioso, así que conversamos con ellos, se les educa, porque ellos son quienes van a continuar con el cuidado en casa..." (Licenciada)

Categoría II: Atributos De La Práctica De Enfermería Frente Al Entorno Del Adulto

Mayor.

2.1. El cuidado al adulto mayor y las limitaciones.

Cuidar es ante todo un acto de vida, significa movilizar las capacidades del ser humano en su cotidianidad, en el proceso de vida muerte, en estado de salud o enfermedad, teniendo en cuenta sus costumbres, con el objeto de lograr el desarrollo de su capacidad de vivir o de compensar 
y suplir la alteración de sus funciones biosociales, implica acercarse al otro con una actitud ética de apertura, de sensibilidad y de responsabilidad ante las experiencias propias y de los otros orientando la aplicación de sus conocimientos y habilidades.

Ante lo expuesto anteriormente y comparándolo con la información obtenida a través de las entrevistas, las enfermeras manifiestan.

“...Me gusta atender a los adultos mayores, he trabajado en otros servicios donde la mayoría eran adultos, si haces algo que te gusta lo vas a realizar con mucho ánimo y dedicación, así tengas 10 pacientes para cuidar, haciendo el turno muy ameno..." (Cuidadora del adulto)

\subsection{Atributos de la práctica de enfermería bajo}

la mirada CIPE.

El CIPEconsidera entre su clasificación tres femémonos importantes para el cuidado del adulto mayor como son: los fenómenos fisiológicos, esenciales para la supervivencia del ser humano, del cual se deprenden las principales necesidades como la respiración, termorregulación, alimentación, entre otras, dando un orden a las actividades de enfermería, posterior a estos se continua con la valoración de los fenómenos psicológicos y del entorno, importantes de valorar, según el CIPE y por las enfermeras para que el cuidado sea de manera humanizada.

Ante lo expuesto anteriormente y comparándolo con la información obtenida a través de las entrevistas, las enfermeras manifiestan.

\section{“...Empezamos por vías respiratorias que es una} necesidad básica: nebulizamos, limpiamos secreciones orales y bronquiales, aspiramos. Ante de utilizar el aspirador realizamos la extracción manual, evitando lacerar la cavidad bucal..." (Cuidadora)

Es así que la primera necesidad que se consideran como principal para el cuidado del adulto mayor es la respiración, considerando además las actividades que realizan para valorar esta necesidad. El monitoreo de las constantes vitales es prioridad en el cuidado del adulto mayor, considerando a la presión arterial como la más resaltante.

“...En caso de la frecuencia cardiaca vemos pacientes taquicardicos, entonces valoramos su ritmo y frecuencia a la par Valoramos la temperatura, porque normalmente cuando hay una fiebrela frecuencia cardiaca aumenta..." (Trabajadora de salud)

Es por eso que la valoración de la frecuencia cardiaca en necesario para detectar si existe o no alteración de sus principales características. Al comparar esto con los datos cuantitativos, obtenemos que las enfermeras pongan más énfasis en valorar el ritmo y la frecuencia representado por el $57 \%$ siempre, seguida de la valoración de la profundidad en un $43 \%$ que siempre lo valoran. Obteniendo que las enfermeras valoran la función cardiaca con las actividades que propone el CIPE, aunque no en igual proporción, pero se rigen en esas actividades.

En la necesidad de Mantener la temperatura corporal, nos manifiestan que es valorada cuando está alterada, por una enfermedad concomitante, o por el mismo el proceso de envejecimiento.

Ante lo expuesto anteriormente y comparándolo con la información obtenida a través de las entrevistas, las enfermeras manifiestan.

“...En la necesidad de nutrición, la valoramos si el paciente esta desnutrido, anémico, mastica, deglute, evaluamos y reportamos al médico, para poder colocar una sonda nasogástrica, vemos que consuma toda su dieta, y se finaliza con la valoración de la sonda colocada, el tiempo de cambio, la alimentación, educación al familiar o cuidador, se coordina con nutrición, el peso del 
paciente, su contextura, supervisamos que consuma su dieta..." (Licenciada)

La valoración que realizan los sujetos de investigación frente a esta necesidad, se sustenta en relación a su alteración rigiéndose de las órdenes médicas para ejecutar sus procedimientos.

“...Eliminación urinaria verificamos el tipo sonda para realizar el cambio respectivo, considerar a los pacientes que tiene retención urinaria, si usan sonda, presencia de pañal o colector, verificamos si existe alteración dermatológica como las escaldaduras, cambiando oportunamente el pañal, además de valorar las características de la orina, casi siempre..." (Cuidadora)

Es por eso que la valoración que realiza la enfermera frente a la alteración de esta necesidad debe ser de manera completa.

“...En las necesidades fisiológicas, la integridad de la piel, porque se pierde la hidratación, volviéndose más propensa a una lesión, eso es lo principal en nuestro adulto mayor..." (Enfermera)

Se considera la necesidad de la piel como una de las prioritarias a valorar en el cuidado del adulto mayor por el mismo proceso de envejecimiento al que se afrontan.

“... También valoramos la necesidad de sueño, si un paciente no puede dormir quizás por una mala posición o porque hay mucho ruido en el ambiente, entonces a acá es donde se identifica que factores están alterando su sueño..." (Trabajadora de salud)

Pues la valoración completa e integra de esta necesidad permite detectar si existen o no factores que provoquen su alteración, generando incomodidad en el adulto mayor.

“...Algunos pacientes son de grado 3 o 4, no pueden bajarse por sí solos de la cama, se pueden caer, entonces tenemos que estar poniendo las barandas, si el familiar no se encuentra..." (Cuidadora)

Las actividades de enfermería para la necesidad de movimiento, está en relación del grado de dependencia y tipo de paciente, en este caso los adultos mayores.

“...Valoramos mucho el dolor y sus características, sobre todo la intensidad, pacientes que estén con mucho dolor de inmediato se tiene que actuar, de acuerdo a la indicación médica que nosotras acudimos para un tratamiento que es la terapia del dolor, eso es como prioridad. Además del autocuidado..." (Trabajadora de salud)

Siendo lo primordial valorar la presencia de dolor y su intensidad, sin embargo muchas de las enfermeras acuden a la indicación médica para el complemento farmacológico, con la finalidad de satisfacer esta necesidad.

“...En las necesidades psicológicas, en el adulto mayor encontramos con frecuencia la depresión, soledad, los pacientes muchas veces están abandonados, hay maltrato verbal, conflictos familiares, entonces llamamos a la asistenta social, el apoyo espiritual independientemente de su religión, lo recibe del sacerdote que viene a visitarlos, el familiar también está ansioso, así que conversamos con ellos, se les educa, porque ellos son quienes van a continuar con el cuidado en casa..." ( Licenciada)

El trabajo multidisciplinario tiene gran influencia sobre el cuidado que se brinda al adulto, sobre todo en aquellas necesidades donde la enfermera solo puede orientar mas no solucionarlas como las psicológicas, es por eso que creen necesarios derivar al profesional correspondiente, para solucionar problemas presentes en la misma persona $o$ en el familiar, sobre todo en estos últimos ya que son ellos los que continúa con el cuidado en casa, muy aparte de estas cabe destacar al apoyo espiritual. Después de haber analizado todos los resultados obtenido podemos decir que las prácticas de enfermería desde la perspectiva CIPE para el cuidado del adulto mayor, 
significa contar con una clasificación organizada y completa que involucran los principales fenómenos que suceden en la persona, permitiendo realizar una valoración más ordenada y completa, en donde se identifique a la persona en todos sus aspectos, considerándolo como un ser pandimensional, es por eso que esta clasificación permite introducir nuevas actividades de Enfermería para planificar el mejor cuidado, respondiendo a las expectativas de un grupo etario tan susceptible como es el adulto mayor y que muchas veces es desvalorado por las sociedad e incluso por el mismo profesional de salud.

EI CIPE es un modelo de clasificación rescatable para la enfermería geriátrica, pues identifica los principales fenómenos o necesidades de las personas, específicamente del adulto mayor, los cuales debido al propio proceso de envejecimiento, tienden a afrontar cambios tantos fisiológicos como psicológicos que hacen que su salud se vaya deteriorando progresivamente, es por eso que este modelo de clasificación permitirá que el trabajo de enfermería se organice en base a prioridades de necesidades dándole orden a la práctica, para responda de manera inmediata a las necesidades del adulto mayor.

Desde el punto de vista de la Clasificación Internacional para la práctica de Enfermería, las prácticas están organizadas en relación a la persona y su entorno, el primero representado en base a dos fenómenos esenciales para la práctica de enfermería, siendo los fisiológicos y psicológicos los prioritarios en relación al cuidado del adulto mayor. Las enfermeras organizan actividades de cuidado al adulto mayor mediante una clasificación donde se especifican aquellas necesidades consideradas como primordiales para los adultos mayores y por ende importantes de considerar durante la valoración que la Enfermería realiza en cada una de estas actividades consideradas en esta clasificación.

Las prácticas de Enfermería en el adulto mayor actualmente se reflejan en las actividades que realiza la enfermera para su cuidado, actividades que muchas veces implica no seguir una secuencia especifica de lo que se realiza, priorizando algunas veces sus actividades de acuerdo a su criterio, no siendo el mismo para todas, es por eso que muchas de estas actividades no se evidenciadas en la práctica reflejando de esta manera la poca productividad que realiza la enfermera, generando incomodidad en los profesionales, debido a que no se sigue una continuidad del cuidado y no refleja de esta manera las prácticas de enfermería.

Las prácticas de Enfermería son inherentes al cuidado, considerándose estas prácticas como la esencia del actuar de Enfermería dirigido a los adultos mayores, establecidas estas como una relación entre la enfermera y la persona que se cuida, yendo esto más allá de lo que su actuar sea, simplemente cumplir con lo que se indica, reflejándose de esta manera que sus prácticas están amparadas bajo un pensamiento y juicio clínico. Las prácticas de Enfermería, no solo se enfocan en el aspecto fisiológico del adulto mayor, sino también en su aspecto psicológico, que muchas veces en su totalidad no son valorados debido a la misma rutina a las que están acostumbradas las enfermeras o simplemente a la sobrecarga laboral a las que estas sometidas, haciendo que todos estos factores influyan sobre el cuidado que se le brinda al adulto mayor.

En la valoración que las enfermeras realizan de cada uno de los fenómenos que propone el CIPE, se puede identificar que cada necesidad presentar sus propias características, las cuales son consideradas por las enfermeras para valorar las necesidades del adulto mayor, sin embargo muchas de estas características son valoradas tomando en cuenta si se presenta o no una alteración de la necesidad, sin embargo la importancia que le otorga el CIPE a cada una de estas necesidades, es la valoración completa de todo sus aspectos con o sin alteración de la necesidad.

El cuidado del adulto mayor, no solo implica al actuar de enfermería frente a las diferentes necesidades alteradas, sino que va más allá, es decir el trabajo multidisciplinario, como en este caso puede ser la colaboración del psicólogo, la nutricionistas, entre otros, profesionales de la salud, permitiendo que el cuidado puede ser el 
mejor para este tipo de población, logrando su pronto recuperación y por ende el reingreso a la sociedad.

Los significados que le otorga esta clasificación al cuidado del adulto mayor, emergen de actividades que muchas veces no son consideradas durante su valoración, pero necesarias de realizar para reflejar el cuidado de Enfermería, siendo este un aporte para que la práctica de Enfermería se realice de manera profunda, reconociendo en cada una de las necesidades alteradas aquellas características que muy pocas veces son reconocidas, pero necesarias de valorar, de manera que permita a la enfermera tener una idea de cuáles serán las actividades que ser realizan frente a toda la problemática encontrada.

Las enfermeras manifiestan que reconocen cuales son los pacientes más críticos al momento de evaluarlos y valorarlos, sin embargo al observarlas vemos que sus actividades estas más ligadas a sus problemas, lo que genera que solo cuando ven al paciente delicado se preocupan más por valorar a detalle las necesidades fisiológicas del adulto mayor.

La fragmentación del cuidado de una persona adulta mayor, ha generado que las actividades estén relacionadas a los siguientes aspectos: la respiración, como necesidad primordial de todo ser, sobre todo la saturación de oxígeno, como unas de las principales actividades de enfermería. La eliminación urinaria como una de las actividades más frecuentes en los enfermos, será porque se considera que el control de la diuresis es permite liberación de sustancias de desechos, para mantener el buen funcionamiento de los diferentes órganos. La integridad de la piel identificando sobre todo la presencia de lesiones que puedan originar infecciones o enfermedades sobre agregadas que hagan que la recuperación sea más lenta. En la investigación lo que se describa es sistematizar la práctica que la enfermera realiza en beneficio del cuidado del adulto mayor, ya que finalmente la esencia de enfermería es el cuidado.

Con todo esto se evidencia que las prácticas de enfermería generalmente están orientadas a valorar solo las necesidades primordiales es decir las más superiores, dejando de lado muchas veces las necesidades menos valoradas por las enfermeras como son las: la higiene, la alimentación, vestimenta.

\section{Bibliografía}

1. Guillermo R. [Tesis Magistral]. Colombia Medellín ;2009 .Disponible en: http://tesis.udea.edu.co/dspace/bitstrea $\mathrm{m} / 10495 / 787 / 1 /$ FactoresRelacionadosco nlaaplicaci\%C3\%B3n.PDF

2. Kèrouac $S$. et al. El Pensamiento Enfermero. 5 Edición. Barcelona-España: ELSEVIER MASSON; 2007.

3. Diaz DW, Nieto GC. Utilización del proceso de enfermería en el ejercicio clínico profesional en seis instituciones prestadoras de servicios de salud en Santafé Bogotá, (Revista de enfermería) 1998(acceso 17 de mayo del 2012;1(6) Disponible en: http://www.encolombia.com/medicina/en fermeri a/enfermeria3200utilizacion 1.htm

4. Railer A, Marriner A. Modelos y Teorías de Enfermería. 7 Edición. España: ELSEVIER ESPAÑA, S.L.; 2011.

5. Theune B. La Clasificación Internacional para la Práctica de Enfermería como ejemplo de Investigación en Red. SCielo. 2004; 10(2). Acceso: 07 de agosto del $2012 . \quad$ Disponible en: http://www.scielo.cl/scielo.php?pid=S071 $795532004000200002 \&$ script $=$ sci_arttext

6. Cubas R, Honorato da Silva S, Rosso M. Classificação Internacional para a Prática de Enfermagem (CIPE): uma revisão de literatura, International Classification for Nursing Practice (ICNP): a revision of literatura.ClasificaciónInternacional para la Práctica de Enfermería (CIPE): una revisión de literatura. Rev. Eletr. Enf. [Internet]. 2010; 12(1):186-94. 
7. Menga L, Marli A. Pesquisa En Educación. Abordajes Cualitativos. EDU. Sao PauloBrasil; 1986.

8. Rodríguez G, Gil J, García E. Metodología De La Investigación Cualitativa. Maracena: Ediciones Aljibe, S.L.; 1996.

9. Canales F. "Metodología de la Investigación. Manual para eldesarrollo del personal de Salud". $2^{\circ}$ Edición. 1998

10. Sgreccia E. Manuale di Bioetica. ob.cit.Tomo II, p. 491. 2010.

11. Palazzi L. La Fundamentación Personalista En Bioética. [sede Web]* Biblioteca Católica Digital [actualizada el 3 de enero de 2006; acceso 20 de Octubre de 2011]. Disponible en:
http://www.mercaba.org/FICHAS/bioetic/f undamentacion_personalista_en_bioetica.h tm.

12. Vargas M. Sobre El Concepto De La Percepción. México D.F.: Centro De I n for m a c i ó n Y D o c u m e n t a c i ó n Antropológica, Universidad Autónoma Metropolitana-Iztapalapa /Departamento De Atención A La Salud, Universidad Autónoma MetropolitanaXochimilco.1994

13. Kozier B. Fundamentos de enfermería: conceptos, proceso y práctica: Mc GrawHill Interamericana; Madrid, 1994.

14. Potter P, Perry A. fundamentos de enfermería. 5ta ed. España: Harcourt Oceano; 2002 